\title{
REVIEW
}

\section{Pediatric thoracic surgery}

\author{
Michael Hollwarth
}

Accepted: 22 July 2009/Published online: 29 August 2009

(C) Springer-Verlag 2009

Pediatric Thoracic Surgery edited by Parikh, Crabbe, Auldist and Rothenberg is among the latest textbooks devoted to surgical disorders of the thorax. It is a well rounded and comprehensive book covering excellent information about common and rare functional and anatomical problems and diseases of the thorax in neonates, infants and children. The book is well organized, divided into 10 sections and 48 chapters spanning 600 pages. There are a total of 50 contributors of international well-recognized experts in pediatric thoracic surgery.

Each section covers a major subject area such as fundamental considerations, infection, trauma, tumors, esophagus, trachea, lung disease, diaphragm, chest wall abnormalities and miscellaneous. Within the sections, the editors have chosen the most relevant topics. The figures and tables are instructive and appropriate for the chapter content. Some figures are simply hand drawn, but show most excellently the needed information. The editors have done an excellent job in providing up to date information in most chapters that covers current anatomic, physiologic, anesthetic, radiologic, endoscopic and open as well videoassisted surgical techniques used in thoracic diseases. At the beginning, most of the chapters include historical, anatomical and physiological paragraphs relevant to the topic, and they end with appropriate conclusions and further perspectives. The number of references in the chapters varies between 13 and 185. Most of the references-but not all-are up to date even with citation from 2007. The rather brief paragraph about Foregut Septation in the chapter of Developmental Biology is well balanced by the paragraph on the Embryogenesis of Esophageal Atresia in the chapter of Congenital Malformations. The latter is again excellently supported by an additional chapter on the different options of Esophageal Replacement. Unfortunately, the section on the trachea does not include a chapter about the problem of short and long tracheal stenosis and the relevant surgical options.

In conclusion, this textbook is a comprehensive and a very balanced overview of thoracic surgical problems in children. It is informative and provides an excellent overall resource for pediatric surgeons with an interest in thoracic surgery. The editors and contributors provide the reader with the proper medical and surgical principles and the book should prove to be an important reference textbook in this area for the next several years. 